\title{
Asociación de estrategias de afrontamiento y calidad de vida relacionada con la salud en pacientes en diálisis peritoneal
}

\author{
Ana Miriam Cabrera-Delgado ${ }^{1}$, María Cristina Rodríguez-Zamoraㄹ, María de Jesús Ventura-García², Carolina \\ Santillán-Torres ${ }^{1}$, Dante Amato ${ }^{1 *}$ \\ ${ }^{1}$ Facultad de Estudios Superiores Iztacala. Universidad Nacional Autónoma de México. Tlalnepantla. México \\ ${ }^{2}$ Unidad de Investigación Médica en Enfermedades Nefrológicas. Instituto Mexicano del Seguro Social. Ciudad de \\ México. México
}

Como citar este artículo:

Cabrera-Delgado AM, Rodríguez-Zamora MC, Ventura-García MJ, Santillán-Torres C, Amato D. Asociación de estrategias de afrontamiento y calidad de vida relacionada con la salud en pacientes de diálisis peritoneal.

Enferm Nefrol. 2019 Oct-Dic;22(4):398-404

\section{Resumen}

Objetivo: Evaluar la relación entre estrategias de afrontamiento y calidad de vida relacionada con la salud en pacientes incidentes en diálisis peritoneal.

Material y Método: Estudio transversal en 106 pacientes incidentes en diálisis peritoneal, en cuatro centros de Ciudad de México. Se usaron los instrumentos Kidney Disease Quality of Life Short Form (KDQOL-SF36) y Coping Strategies Inventory para evaluar la calidad de vida relacionada con la salud y las estrategias de afrontamiento, respectivamente. Se evaluó la correlación de puntuaciones de estrategias de afrontamiento y calidad de vida. Se comparó la calidad de vida relacionada con la salud entre los pacientes con estrategia de afrontamiento activa y pasiva.

Resultados: La estrategia de afrontamiento activa mostró correlación positiva con componentes físico y mental, efectos de la enfermedad renal y carga de la enfermedad renal del KDQOL-SF36, en tanto que el puntaje de estrategia pasiva correlacionó negativamente con componente mental y carga de la enfermedad renal en el KDQOL-SF36. Los puntajes de componentes físico y mental, efectos de enfermedad renal, carga de enfermedad renal y problemas

\section{Correspondencia:}

Dante Amato

dante.amato@unam.mx por síntomas del KDQ0L-SF36 fueron significativamente más altos en pacientes con estrategia de afrontamiento activa que en los que presentaron estrategia pasiva.

Conclusiones: A partir de los resultados encontrados podemos concluir que los pacientes con estrategia de afrontamiento activa presentan mejor calidad de vida relacionada con la salud, tanto en las dimensiones del cuestionario específico de las alteraciones renales como en el genérico de calidad de vida, en comparación con los pacientes con estrategia de afrontamiento pasiva.

PALABRAS CLAVE: calidad de vida relacionada con la salud; estrategias de afrontamiento; diálisis peritoneal.

Association of coping strategies and healthrelated quality of life in patients on peritoneal dialysis

\section{Abstract}

Background: An important feature of chronic diseases, is it permanent character with a significant impact on long-term treatments, as it is the case of patients on peritoneal dialysis, that transcendently modifying their personal, family and social dynamic.

Objective: To evaluate the relationship of coping strategies and health-related quality of life in incident patients on peritoneal dialysis. 
Material and Method: One hundred and six incident patients (within 0 to 3 months from the beginning of PD treatment) from 4 PD centers in Mexico City were included in a cross-sectional study. Kidney Disease Quality of Life Short Form (KDQOL-SF36) and Coping Strategies Inventory (CSI) were used to evaluate health-related quality of life and coping strategies respectively. Correlation of the coping strategies and the health-related quality of life scores were assessed. Distributions of health-related quality of life scores between engagement and disengagement coping strategies were compared.

Results: Engagement coping strategy score showed significant positive correlation with the KDQOL SF-36 physical and mental composite, effects of kidney disease and burden of kidney disease, while disengagement coping strategy score negatively correlated with the KDQOL SF-36 mental component, and burden of kidney disease. KDQOL SF-36 physical and mental components, effects of kidney disease, burden of kidney disease, and symptom problem scores were significantly higher in patients with engagement coping strategy than in patients with disengagement coping strategy.

Conclusions: The results support the hypothesis of a relationship between coping strategies and healthrelated quality of life.

Keywords: health related quality of life; KDQOL SF36; coping strategies; CSI; peritoneal dialysis.

\section{Introducción}

Un rasgo importante de las enfermedades crónicas, es su carácter permanente con un significado impactante en los tratamientos a largo plazo, como es el caso de los pacientes en diálisis peritoneal, modificando de forma trascendente su dinámica personal, familiar y social ${ }^{1 .}$

La calidad de vida relacionada con la salud (CVRS) se ve afectada en la enfermedad renal crónica avanzada desde la etapa de prediálisis ${ }^{2}$; siendo un predictor independiente de desenlaces clínicos tanto en hemodiálisis $(H D)^{3}$, como en diálisis peritoneal (DP $)^{4}$ Los pacientes con enfermedad renal crónica en Tratamiento Renal Sustitutivo (TRS) tienden a presentar peor CVRS que la población en general "sana"5,6. Por otro lado, en pacientes con cáncer o insuficiencia cardiaca crónica, algunas estrategias de afrontamiento (EA) parecen estar asociadas con mejores desenlaces clínicos, como mortalidad y $\mathrm{CVRS}^{7,8}$. Las estrategias de afrontamiento pueden modificarse mediante terapia de la conducta ${ }^{9,10}$, y éstas parecen ser eficaces para mejorar ciertos síntomas psicológicos en los pacientes en $\mathrm{HD}^{11}$.

Los estilos de afrontamiento son definidos como esfuerzos cognitivo-conductuales para manejar demandas internas o externas que se perciben mayores a los recursos de la persona (independientemente a que se obtenga un resultado exitoso o no $)^{12,13}$ y comprende ocho estilos típicamente descritos ${ }^{14}$ :

1. Resolución de problemas en donde el esfuerzo cognitivo-conductual se enfoca en modificar las situaciones estresantes.

2. Reestructuración cognitiva orientada a resignificar el concepto de estresores.

3. Apoyo emocional caracterizada por la búsqueda de sustento.

4. Expresión emocional encaminada a la liberación de la respuesta al estrés.

5. Evitación de problemas en donde sobresale la negación.

6. Pensamiento desiderativo donde la estrategia radica en el deseo de una realidad menos adversa.

7. Retirada social definida por distanciamiento de redes afectivas.

8. Autocrítica identificada por la autoinculpación del individuo en la incidencia de factores estresantes.

También se ha descrito, en pacientes en DP, que a mayor tiempo de tratamiento disminuyen las $E A^{15}$.

Por todo ello, con la hipótesis conceptual de que los pacientes en DP con una EA activa tienen mejor CVRS que los que tienen una EA pasiva, elaboramos el presente estudio cuyo objetivo fue evaluar la relación entre EA y CVRS en pacientes incidentes en DP. 


\section{Material y método}

\section{Diseño y muestra}

Se hizo un estudio observacional descriptivo de corte transversal en el que participaron 106 pacientes adultos con enfermedad renal crónica en TRS con DP en la Ciudad de México, en instalaciones del Instituto Mexicano del Seguro Social (IMSS) o el Instituto de Seguridad y Servicios Sociales de los Trabajadores del Estado (ISSSTE); con los siguientes criterios de inclusión:

-Mayores de 18 años.

-Pacientes incidentes (entre 0 y 3 meses desde el inicio del tratamiento con DP).

\section{-Clínicamente estables.}

\section{Variables de estudio e Instrumentos de medida}

Se estudiaron la CVRS y las EA. Para el análisis de la CVRS y las EA, se utilizaron las versiones en español de los cuestionarios Kidney Disease Quality of Life Short Form (KDQOL SF-36, versión 1.3) y Coping Strategies Inventory (CSI), respectivamente, validados en poblaciones mexicanas ${ }^{4,16,17}$. Ambos cuestionarios se aplicaron en la misma sesión.

El cuestionario KDQOL combina el instrumento genérico SF-36 con otro específico para enfermedad renal. Los resultados del instrumento SF-36 se resumen en componente físico (CF) y componente mental (CM) mediante el algoritmo de calificación de RAND ${ }^{18}$. EI CF incluye las dimensiones de funcionamiento físico, rol físico, dolor corporal y también incluye elementos de salud general, vitalidad y funcionamiento social, medidos en una escala de 100 puntos (cuanto más alta sea la calificación, mejor es la CVRS del paciente). En la población general, la media para cada una de las dos escalas (CF y CM) es de 50 puntos, con una desviación estándar (DE) de 10 puntos $^{3}$. En este trabajo se incluyen además tres dominios relacionados con enfermedad renal del componente específico para la enfermedad del instrumento KDQOL, también calificados en una escala de 100 puntos: 1) carga de enfermedad renal, 2) efectos de la enfermedad renal y 3) problemas por síntomas.

En el CSI las EA se categorizan en activa y pasiva. Estas dos categorías a su vez se subdividen en estrategia enfocada en el problema y enfocada en las emociones. La estructura de CSI comprende las siguientes cuatro subcategorías: activa enfocada en el problema, pasiva enfocada en el problema, activa enfocada en emociones y pasiva enfocada en emociones. Para cada subcategoría hay cuatro reactivos que se califican mediante una escala de Likert en la que $1=$ nunca, $2=a$ menudo, $3=$ rara vez y $4=$ casi siempre. La puntuación en cada subcategoría se calcula con la suma del puntaje de todos los reactivos pertenecientes a ella y puede ir de 4 a 20. Cada subcategoría incluye dos dimensiones: solución de problemas y reestructuración cognitiva en activa enfocada en el problema, evitación de problemas y pensamiento desiderativo en pasiva enfocada en el problema, expresión emocional y apoyo social en activa enfocada en emociones y autocrítica y retirada social en pasiva enfocada en emociones ${ }^{19}$. Para el análisis que aquí se presenta, los pacientes se clasificaron como pertenecientes a la categoría de estrategia activa 0 pasiva ${ }^{14}$. También se registraron algunas variables sociodemográficas (edad, sexo, estado civil, nivel educativo, estatus laboral, ingreso económico y redes de apoyo social) y clínicas (tiempo en DP, albúmina y creatinina séricas, tensión arterial y comorbilidades).

\section{Aspectos/consideraciones éticas}

Los comités de investigación y ética de la Facultad de Estudios Superiores Iztacala, IMSS e ISSSTE aprobaron el protocolo del estudio. Todos los pacientes accedieron voluntariamente a participar y firmaron un formato de consentimiento informado.

\section{Análisis estadístico}

Las variables cuantitativas se presentan como la media \pm desviación estándar (DE), las puntuaciones del KDQ0L SF-36 como mediana e intervalo intercuartílico y las variables categóricas como frecuencias. Para el análisis de correlación entre las EA y la CVRS se utilizó el coeficiente de correlación por rangos de Spearman. La comparación de las distribuciones de las puntuaciones de la CVRS entre las diferentes EA se hizo con la prueba $\mathrm{U}$ de Mann-Whitney. Todas las pruebas estadísticas se hicieron con SPSS versión 21. Los valores de $p<0,05$ se consideraron estadísticamente significativos.

\section{Resultados}

Las características sociodemográficas y clínicas de los pacientes estudiados se presentan en la Tabla $\mathbf{l}$. 
Tabla 1. Características sociodemográficas y clínicas de los pacientes estudiados.

\begin{tabular}{|l|l|}
\hline Edad (años) & $59,6 \pm 11,2$ \\
\hline Sexo (n, M/F) & $67 / 39$ \\
\hline Peso (kg) & $67,6 \pm 14,8$ \\
\hline Estatura (m) & $1,6 \pm 0,1$ \\
\hline Creatinina sérica (mg/dl) & $7,5 \pm 2,3$ \\
\hline Albúmina sérica (g/dl) & $3,0 \pm 0,5$ \\
\hline Tensión arterial sistólica (mm Hg) & $130,3 \pm 22,5$ \\
\hline Tensión arterial diastólica (mm Hg) & $73,6 \pm 10,4$ \\
\hline Diabetes mellitus (n) & 87 \\
\hline Hipertensión (n) & 92 \\
\hline Tiempo en DP (meses) & $1,6 \pm 0,7$ \\
\hline DPCA/DPA (n) & $84 / 22$ \\
\hline IMSS/ISSSTE (n) & $54 / 52$ \\
\hline Cónyuge o pareja ( $n$, si/no) & $77 / 29$ \\
\hline Nivel educativo $(n, \leq$ primaria/> primaria) & $68 / 38$ \\
\hline Estatus laboral $(n$, empleado/desempleado) & $33 / 73$ \\
\hline
\end{tabular}

Los valores son medias \pm desviaciones estándar o frecuencias. M: masculino. F: femenino. DP: diálisis peritoneal. DPCA: diálisis peritoneal continua ambulatoria. APD: diálisis peritoneal automatizada. IMSS: Instituto Mexicano del Seguro Social. ISSSTE: Instituto de Seguridad y Servicios Sociales de los Trabajadores del Estado.

En la Tabla 2, están representados los valores de la CVRS del total de pacientes de la muestra.

Tabla 2. Puntuaciones obtenidas en las cinco escalas de CVRS

\begin{tabular}{|l|l|}
\hline Dimensiones CVRS & Puntuaciones \\
\hline $\begin{array}{l}\text { Lista de síntomas y problemas } \\
\text { Puntuación mínima } \\
\text { Mediana } \\
\text { Puntuación máxima }\end{array}$ & 47,91 \\
\hline Efectos de la enfermedad renal & 83,33 \\
\hline Puntuación mínima & 100 \\
\hline Mediana & \\
\hline Puntuación máxima & 12 \\
\hline Carga de la enfermedad renal & 68,75 \\
\hline Puntuación mínima & 100 \\
\hline $\begin{array}{l}\text { Mediana } \\
\text { Puntuación máxima }\end{array}$ & 0 \\
\hline Componente físico & 40,62 \\
\hline Puntuación mínima & 93,75 \\
\hline Mediana & \\
\hline Puntuación máxima & 16,50 \\
\hline Componente mental & 41,77 \\
\hline $\begin{array}{l}\text { Puntuación mínima } \\
\text { Mediana }\end{array}$ & 59,31 \\
\hline Puntuación máxima & \\
\hline
\end{tabular}

Las puntuaciones de las EA de compromiso mostraron una correlación significativa positiva con los componentes físico y mental y la carga de enfermedad renal del KDQOL SF-36 (Tabla 3).

Tabla 3. Correlación entre los puntajes de KDQOL SF-36 y CSI.

\begin{tabular}{|c|c|c|}
\hline & Activo & Pasivo \\
\hline \multicolumn{3}{|l|}{ SF-36 } \\
\hline Resumen componente físico & $0,232(0,01)^{*}$ & $0,021(0,419)$ \\
\hline Resumen componente mental & $0,176(0,04)^{*}$ & $-0,189(0,036)^{*}$ \\
\hline \multicolumn{3}{|l|}{ KDQOL } \\
\hline Carga de enfermedad renal & $0,267(0,004) * *$ & $-0,266(0,004) * *$ \\
\hline Efectos de enfermedad renal & $0,238(0,009)$ * * & $-0,072(0,238)$ \\
\hline Problemas por síntomas & $0,140(0,082)$ & $-0,154(0,063)$ \\
\hline
\end{tabular}

Los valores son el coeficiente ro de Spearman y $(p) .{ }^{*} p<0,05 ;{ }^{* *} p<0,01$.

Las puntuaciones de los componentes físico y mental, efectos de la enfermedad renal, carga de la enfermedad renal y problemas por síntomas fueron significativamente más altos en los pacientes con EA de compromiso que en los que tenían EA de ausencia de compromiso (Tabla 4).

No se encontraron diferencias significativas en la CVRS respecto al sexo, edad, tiempo en diálisis, modalidad de DP (DPCA o DPA), institución (IMSS o ISSSTE), estado civil, nivel educativo o estatus laboral.

\section{Discusión}

El objetivo del presente estudio fue evaluar la relación entre EA y CVRS en pacientes incidentes en DP de la Ciudad de México. Como principal resultado de este estudio, podemos destacar que las puntuaciones de la CVRS son significativamente más altas en los pacientes con EA activa que en los que tenían EA pasiva. Además, los pacientes con puntuaciones más altas del KDQOL SF-36 también tuvieron puntuaciones más altas en la escala EA activa del CSI. Además, las puntuaciones más bajas en la escala del KDQOL SF-36 mostraron una tendencia no significativa a estar asociados con puntuaciones más altas en la escala de EA pasiva del CSI. Estos resultados concuerdan con observaciones previamente publicadas en pacientes con cáncer ${ }^{20,21}$, insuficiencia cardiaca ${ }^{8,22}$, esclerosis múltiple ${ }^{23}$, enfermedad cerebrovascular ${ }^{24}$ y síndrome de colon irritable ${ }^{25}$, lo que apoya la hipótesis de que las EA activa y orientada al problema se asocia con mejor CVRS y la EA pasiva y orientada a las emociones tiende a relacionarse con peor CVRS. 
Tabla 4. Comparación de los puntajes de KDQOL SF-36 entre diferentes estrategias de afrontamiento.

\begin{tabular}{|c|c|c|c|}
\hline & Activa $(n=65)$ & Pasiva $(n=41)$ & $p$ \\
\hline $\begin{array}{l}\text { SF-36 } \\
\text { Resumen componente físico } \\
\text { Resumen componente mental }\end{array}$ & $\begin{array}{l}44,5(36,4-51,2) \\
44,5(36,1-51,4)\end{array}$ & $\begin{array}{l}39,0(31,1-45,8) \\
41,6(30,0-47,1)\end{array}$ & $\begin{array}{l}0,037^{*} \\
0,021^{*}\end{array}$ \\
\hline $\begin{array}{l}\text { KDQOL } \\
\text { Carga de enfermedad renal } \\
\text { Efectos de enfermedad renal } \\
\text { Problemas por síntomas }\end{array}$ & $\begin{array}{l}43,8(31,3-62,5) \\
78,1(59,4-90,6) \\
85,4(70,8-91,7)\end{array}$ & $\begin{array}{l}31,3(12,5-50,0) \\
62,5(51,6-75,0) \\
79,2(62,5-88,5)\end{array}$ & $\begin{array}{l}0,0001^{* *} \\
0,003 * * \\
0,044^{*}\end{array}$ \\
\hline
\end{tabular}

Los valores son mediana (intervalo intercuartílico). La prueba de hipótesis usada fue la prueba U de Mann-Whitney. * $p<0,05 ;{ }^{* *} p<0,01$.

En general, la mayoría de los estudios muestran, que los pacientes en TRS presentan peores puntuaciones en la CVRS, utilizando el instrumento KDQOL SF ${ }^{26}$. Pedregal-Vega evaluó a poblaciones que diferían proporcionalmente en sus variables sociodemográficas, como por ejemplo, mayor proporción de mujeres o distribución mayor en un rango de edad específico, y sin embargo, la mayoría de los pacientes refirieron mala calidad de vida $(65,4 \%)$, lo cual coincide, en tres de las cinco dimensiones evaluadas con nuestro estudio (carga de la enfermedad renal 41,62, componente físico 41,48 y componente mental 42,23) $)^{27}$.

Respecto a las EA, un estudio observacional longitudinal con una muestra amplia, grande mostró que la EA activa orientada al problema se asocia con supervivencia más prolongada y mejoría de la CVRS en pacientes en HD crónica; sin embargo, dado el diseño observacional del estudio, no pudo valorarse la causalidad ${ }^{28}$.

La relación de EA y CVRS tiene la potencialidad de ser muy importante para la práctica clínica. Se acepta que la CVRS es un predictor independiente de la supervivencia y otros desenlaces relevantes en pacientes con ERET en terapia de reemplazo renal ${ }^{3,4,28}$. Se ha informado que la EA puede modificarse mediante intervenciones como la terapia cognitivo conductual ${ }^{9,10}$. Sin embargo, hasta donde sabemos, no hay estudios publicados en los que se evalúe la modificación de la EA mediante intervenciones en la CVRS en pacientes con enfermedad renal. Por lo tanto, se necesita investigación para poner a prueba el efecto de intervenciones dirigidas a modificar el EA sobre la CVRS y otros desenlaces clínicamente relevantes, como supervivencia y complicaciones en pacientes en diálisis. La principal fortaleza de este estudio es que se evitó el sesgo de anticipación (lead time bias) mediante la inclusión exclusiva de pacientes incidentes.
En cuanto a las características sociodemográficas de la muestra analizada, encontramos mayor proporción de hombres, laboralmente inactivos, con un nivel económico medio, escolaridad mayor a nivel secundaria y grupo etario ubicado entre los 41 y 64 años de edad, resultados similares a los obtenidos por Isidro-Cano ${ }^{29}$.

A partir de los resultados encontrados podemos concluir que los pacientes con EA activa presentan mejor CVRS, tanto en las dimensiones del cuestionario específico de las alteraciones renales como en el genérico de calidad de vida, en comparación con los pacientes con EA pasiva. Se requieren estudios adicionales para evaluar el efecto de intervenciones diseñadas para modificar la EA sobre la CVRS y otras variables clínicamente relevantes en pacientes en DP.

\section{Agradecimientos}

A los pacientes participantes en la investigación por su colaboración.

Al personal médico y enfermería de los Servicios de los servicios de diálisis peritoneal.

\author{
Recibido: 17-08-19 \\ Revisado: 25-08-19 \\ Modificado: 05-09-19 \\ Aceptado: 15-09-19
}




\section{Bibliografía}

1. Tak YR, McCubbin M. Family stress, perceived social support and coping following the diagnosis of a child's congenital heart disease. J Adv Nurs. 2002; 39(2):190-8.

2. Garrido-Blanco R, Arroyo-Priego E, Arana-Ruiz AI, López-Zamorano MD, Tierno C, Crespo R. Calidad de vida y enfermedad renal crónica avanzada. Influencia del aclaramiento renal. Enferm Nefrol 2018;21(4):359-67.

3. Osthus B, Preljevic VT, Sandvik L, Leivestad T, Nordhus IH, Dammen T, et al. Mortality and health-related quality of life in prevalent dialysis patients: Comparison between 12-items and 36-items short-form health survey. Health Qual Life Outcomes. 2012;10:46.

4. Paniagua $R$, Amato $D$, Vonesh E, Guo A, Mujais $S$, Group MNCS. Health-related quality of life predicts outcomes but is not affected by peritoneal clearance: The ADEMEX trial. Kidney Int. 2005;67(3):1093104.

5. Cleary J, Drennan J. Quality of life of patients on haemodialysis for end-stage renal disease. J Adv Nurs. 2005;51(6):577-86.

6. Boateng EA, East L. The impact of dialysis modality on quality of life: a systematic review. J Ren Care. 2011;37(4):190-200.

7. Svensson $T$, Inoue $M$, Sawada $N$, Charvat $H$, Iwasaki $M$, Sasazuki S, et al. Coping strategies and cancer incidence and mortality: The Japan Public Health Center-based prospective study. Cancer Epidemiol. 2016;40:126-33.

8. Graven LJ, Grant JS. Coping and health-related quality of life in individuals with heart failure: an integrative review. Heart Lung. 2013;42(3):183-94.

9. Yalcin BM, Unal M, Pirdal H, Karahan TF. Effects of an anger management and stress control program on smoking cessation: a randomized controlled trial. J Am Board Fam Med. 2014;27(5):645-60.

10. Iioka $Y$, Komatsu H. Effectiveness of a stress management program to enhance perimenopausal wo- men's ability to cope with stress. Jpn J Nurs Sci. 2015;12(1):1-17.

11. Cukor D, Ver Halen N, Asher DR, Coplan JD, Weedon $\mathrm{J}$, Wyka KE, et al. Psychosocial intervention improves depression, quality of life, and fluid adherence in hemodialysis. J Am Soc Nephrol. 2014;25(1):196-206.

12. Folkman S, Lazarus RS. If it changes it must be a process: study of emotion and coping during three stages of a college examination. J Pers Soc Psychol. 1985;48(1):150-70.

13. Folkman S, Lazarus RS, Dunkel-Schetter C, DeLongis A, Gruen RJ. Dynamics of a stressful encounter: cognitive appraisal, coping, and encounter outcomes. J Pers Soc Psychol. 1986;50(5):992-1003.

14. Cano-García FJ, Rodríguez-Franco L, García-Martínez J. Spanish version of the Coping Strategies Inventory. Actas Esp Psiquiatr. 2007;35(1):29-39.

15. Ruiz de Alegría $B$, Basabe-Barañano N, Fernández-Prado E, Baños-Baños C, Nogales MA, Echebarri $M$ y cols. Cambios en las estrategias de afrontamiento en los pacientes de diálisis a lo largo del tiempo. Rev Soc Esp Enferm Nefrol 2009;12(1): 11-7.

16. Dehesa-López $E$, Correa-Rotter $R$, Olvera-CastiIlo D, González-Parra C, Baizabal-0larte R, Orozco-Vega R. Transcultural adaptation and validation of the Mexican version of the kidney disease questionnaire KDQ0L-SF36 version 1.3. Qual Life Res. 2017;26(1):193-8.

17. Rodríguez-Díaz F. J., Estrada-Pineda C., Rodríguez-Franco L., C. B-M. Adaptación del Inventario de Estrategias de Afrontamiento (CSI) a la Población Penitenciaria de Mexico. Psicologia: Reflexão e Crítica. 2014;27(3):415-23.

18. Hays RD, Kallich JD, Mapes DL, Coons SJ, Amin N., Carter W.B., et al. Kidney Disease Quality of Life-Short Form (KDQOL-SF36), Version 1.3: A manual for use and scoring. 1.3 ed. Santa Monica, CA: RAND; 1997. p. 39.

19. Tobin DL, Holroyd KA, Reynolds RV, Wigal JK. The hierarchical factor structure of the coping strategies inventory. Cognitive Therapy and Research. 1989;13(4):343-61. 
20. Nipp RD, El-Jawahri A, Fishbein JN, Eusebio J, Stagl JM, Gallagher ER, et al. The relationship between coping strategies, quality of life, and mood in patients with incurable cancer. Cancer. 2016;122(13):2110-6.

21. Bonnaud-Antignac $A$, Bourdon $M$, Dréno $B$, Quéreux G. Coping Strategies at the Time of Diagnosis and Quality of Life 2 Years Later: A Study in Primary Cutaneous Melanoma Patients. Cancer Nurs. 2017;40(1):E45-E53.

22. Nahlen Bose $C$, Bjorling G, Elfstrom $M L$, Persson $H$, Saboonchi F. Assessment of Coping Strategies and Their Associations With Health Related Quality of Life in Patients With Chronic Heart Failure: the Brief COPE Restructured. Cardiol Res. 2015;6(2):239-48.

23. Farran N, Ammar D, Darwish H. Quality of life and coping strategies in Lebanese Multiple Sclerosis patients: A pilot study. Mult Scler Relat Disord. 2016;6:21-7.

24. Lo Buono V, Corallo F, Bramanti P, Marino S. Coping strategies and health-related quality of life after stroke. J Health Psychol. 2017;22(1):16-28.

25. Stanculete MF, Matu S, Pojoga C, Dumitrascu DL. Coping strategies and irrational beliefs as mediators of the health-related quality of life impairments in irritable bowel syndrome. J Gastrointestin Liver Dis. 2015;24(2):159-64.
26. Rebollo-Rubio A, Morales-Asencio JM, Pons-Raventos ME, Mansilla-Francisco JJ. Review of studies on health related quality of life in patients with advanced chronic kidney disease in Spain. Nefrologia. 2015;35(1):92-109.

27. Pedregal-Vega J. Calidad de vida en el paciente con insuficiencia renal crónica KDOQI-5 en diálisis peritoneal del HGZ/UMF No. 8. "Dr. Gilberto Flores Izquierdo". [Tesis de especialidad en Medicina Familiar]. México: UNAM Posgrado; 2013.

28. Niihata K, Fukuma S, Akizawa T, Fukuhara S. Association of coping strategies with mortality and health-related quality of life in hemodialysis patients: The Japan Dialysis Outcomes and Practice Patterns Study. PLoS One. 2017;12(7):e0180498.

29. Isidro-Cano JF. Asociación entre calidad de vida, el apoyo familiar y los mecanismos de afrontamiento en pacientes con ERC en diálisis peritoneal del HGZ $\mathrm{N}^{\circ} 24$. [Tesis de especialidad en Medicina de Familia]. México: UNAM Posgrado; 2013.

Este artículo se distribuye bajo una Licencia Creative Commons Atribución-NoComercial 4.0 Internacional. https://creativecommons.org/licenses/by-nc/4.0/ 